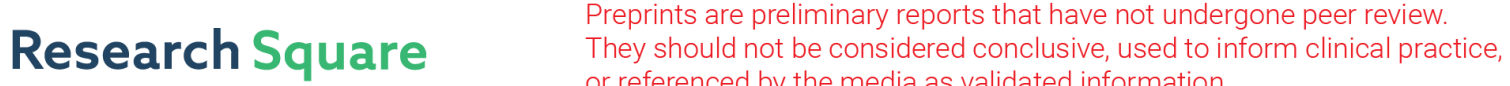 or referenced by the media as validated information. \\ Early Bearing Fault Diagnosis based on the Improved Singular Value Decomposition Method
}

\author{
Lingli Cui \\ Beijing University of Technology \\ Mengxin Sun (D931736607@qq.com ) \\ Beijing University of Technology \\ Jinfeng Huang \\ Beijing University of Technology
}

\section{Research Article}

Keywords: singular value decomposition, fault frequency amplitude, fault information content index, rolling bearing

Posted Date: September 21st, 2021

DOI: https://doi.org/10.21203/rs.3.rs-911468/v1

License: (c) (i) This work is licensed under a Creative Commons Attribution 4.0 International License. Read Full License 


\title{
Early bearing fault diagnosis based on the improved singular value decomposition method
}

\author{
CUI Lingli ${ }^{1}$ SUN Mengxin ${ }^{1}$ HUANG Jinfeng ${ }^{1}$ \\ (1. Faculty of Materials and Manufacturing, Beijing University of Technology, Beijing 100124) \\ E-mail: cuilingli@bjut.edu.cn
}

\begin{abstract}
The traditional singular value decomposition (SVD) method is unable to diagnose the weak fault feature of bearings effectively, which means, it is difficult to retain the effective singular components (SCs). Therefore, a new singular value decomposition method, SVD based on the FIC (fault information content), is proposed, which takes the amplitude characteristics of fault feature frequency as the selection index FIC of singular components. Firstly, the Hankel matrix of the original signal is constructed and SVD is applied in the matrix. Secondly, the proposed index FIC is used to evaluate the information of the decomposed SCs. Finally, the SCs with fault information are selected and added to obtain the denoised signal. The results of bearing fault simulation signals and experimental signals show that compared with the traditional differential singular value decomposition (DS-SVD), the proposed method can select the singular components with larger amount of fault information, and is able to diagnose the fault under the heavy noise interference. The new method can be used for signal denoising and weak fault feature extraction.
\end{abstract}

Keywords: singular value decomposition; fault frequency amplitude; fault information content index; rolling bearing.

\section{Introduction}

As a key component of rotating machinery ${ }^{[1]}$, rolling bearings are directly related to the operation of mechanical equipment and parts and instruments ${ }^{[2]}$. In the light of this, it affects the normal operation of the machine or components, but in the worst case, it will not only cause severe economic losses and even catastrophic personal safety accidents. Therefore, the monitoring and fault identification of bearing operating conditions are particularly significant, which have gradually become one of the research focuses and hotspots ${ }^{[3]}$.

In order to better remove noises and extract features of bearing fault signals, in the past few decades, a variety of signal processing techniques have been developed, including wavelet transform (WT), empirical mode decomposition (EMD), spectral kurtosis (SK), minimum entropy deconvolution, and stochastic resonance. Among them, the singular value decomposition (SVD) method doesn't require pre-defined basis functions, can reveal the weak eigenmodes hidden in the signal and suppress the noise of different distributions, which has attracted a lot of attention of researchers. The existing SVD-based noise reduction methods are usually based on finding a suitable threshold to reconstruct the low-rank matrix for the subsequent processing ${ }^{[4]}$. That is, find a suitable threshold, and then reconstruct the matrix based on this threshold for the subsequent processing.

However, in the case of weak bearing faults, the traditional differential singular value decomposition noise reduction method (DS-SVD) can't find the correct threshold well. In order to solve the above problem, Yang et al. ${ }^{[5]}$ used singular entropy to select the number of effective singular values, when using the singular value decomposition method to denoise the vibration signal. That is, select the order close to saturation as the denoising order and get the noise reduction result with this order. Zhao et al. ${ }^{[6]}$ proposed a new index, using the period modulation degree (PMI) as the criterion for selecting the threshold. By combining singular value decomposition and related kurtosis, Zhang et al. ${ }^{[7]}$ took the kurtosis of the singular component as the basis for selection.

Based on previous studies, a new criterion is proposed in this paper. That is, after the Hankel matrix construction and SVD decomposition of the original signal, the SCs index FIC (fault inclusion degree) is evaluated and calculated. Then the singular component with a large amount of information 
is selected to reconstruct the signal. The analysis results of the simulation signal and the actual rotor vibration signal verify the correctness and superiority of the method, and the weak fault identification of the bearing can be realized.

The arrangement of this paper is as follows: section 2 introduces the basic principles of the SVD noise reduction and the construction method of Hankel matrix; section 3 introduces the new indicator FIC, and elaborates specific steps of the proposed method FIC-SVD; section 4 builds a bearing fault simulation signal and compares the proposed method with the traditional method; section 5 applies this method to actual bearing test data; section 6 draws a conclusion.

\section{The principle of SVD}

For an $m \times n$ matrix, its singular value decomposition is as follows:

$$
A=U \Lambda V^{T}
$$

In the formula, $U=\left[u_{1}, u_{2}, \ldots, u_{m}\right] \in R^{m \times m}$ and $V=\left[v_{1}, v_{2}, \ldots, v_{n}\right] \in R^{n \times n}$ are orthogonal matrices. $u_{i}$ and $v_{i}$ are the left singular vector and the right singular vector, respectively. The column vectors of $U$ and $V$ are the orthogonal eigenvectors of $A A^{\tau}$ and $A^{\tau} A$, respectively. $\Lambda$ is the diagonal matrix storing singular values of the matrix $\mathrm{A}$ in descending order, that is, $\Lambda=$ $\left[\operatorname{diag}\left(\sigma_{1}, \sigma_{2}, \ldots, \sigma_{l}\right), 0\right]$, Where $l=\min (m, n)$ is the rank of the matrix A, and $\sigma_{1} \geq \sigma_{2} \geq \ldots \geq \sigma_{l} \geq$ 0 .

The following describes the process of removing noise from the signal. First, the Hankel matrix is constructed. For the measured discrete signal $X=[x(1), x(2), \ldots, x(N)]$, the following Hankel matrix can be constructed:

$$
A=\left[\begin{array}{cccc}
x(1) & x(2) & \ldots & x(n) \\
x(2) & x(3) & \ldots & x(n+1) \\
\ldots & \ldots & \ldots & \ldots \\
x(m) & x(m+1) & \ldots & x(m+n-1)
\end{array}\right]
$$

It can be seen that $m=N-n+1$, which is used to determine the number of decomposed components in SVD, and it is usually less than $n$.

After that, the signal is subjected to singular value decomposition. According to equation (1), the Hankel matrix A can be expressed as the following equation:

$$
\begin{gathered}
A=\left[u_{1}, u_{2}, \ldots, u_{m}\right]\left[\begin{array}{ccccc}
\sigma_{1} & 0 & \ldots & 0 & 0 \\
0 & \sigma_{2} & \ldots & 0 & 0 \\
\ldots & \ldots & \ldots & \ldots & 0 \\
0 & 0 & \ldots & \sigma_{m} & 0
\end{array}\right]\left[\begin{array}{c}
v_{1}^{\tau} \\
v_{2}^{\tau} \\
\ldots \\
v_{n}^{\tau}
\end{array}\right] \\
=\sigma_{1} u_{1} v_{1}+\sigma_{2} u_{2} v_{2}+\cdots+\sigma_{m} u_{m} v_{m}=A_{1}+A_{2}+\cdots+A_{m}
\end{gathered}
$$

where $u_{i}$ represents the $i$-th column vector of the left singular matrix $\mathrm{U}, u_{i}$ represents the $i$-th column vector of the right singular matrix V. Each matrix $A_{i}$ corresponds to the singular component (SC) $x_{i}$ in the original signal.

The direct method shown in Figure 1 can be used to obtain $x_{i}$ from the matrix $A_{i}$, which is expressed as:

$$
x_{i}=\left[R_{i, 1}, C_{i, n}^{\tau}\right] ; R_{i, 1} \in R^{1 \times n}, C_{i, n} \in R^{(m-1) \times 1}
$$

In the formula, $R_{i, 1}$ is the first row vector of $A_{i}$, and $C_{i, n}$ is the last column vector of $A_{i}$ with the first element removed.

$$
A_{i}=\left[\begin{array}{ccccc}
x_{i}(1) & x_{i}(2) & x_{i}(3) & \cdots & x_{i}(n) \\
\hdashline x_{i}(2) & x_{i}(3) & x_{i}(4) & \cdots & x_{i}(n+1) \\
x_{i}(3) & x_{i}(4) & x_{i}(5) & \ldots & x_{i}(n+2) \\
\ldots & \ldots & \ldots & \cdots & \ldots \\
x_{i}(m) & x_{i}(m+1) & x_{i}(m+2) & \ldots & x_{i}(N) \\
& & & & C_{i, n}
\end{array}\right] R_{i, 1}
$$


Fig.1 Direct method of extracting $x_{i}$ from $A_{i}$

If all the $x_{i}(\mathrm{SCs})$ obtained by the decomposition and reconstruction are added together, the original signal can be well reconstructed.

$$
x=\sum_{i=1}^{m} x_{i}
$$

The traditional method is to denoise the original signal by selecting an appropriate threshold $\mathrm{K}$, and then retaining the SCs corresponding to the top K largest singular values, namely:

$$
\tilde{x}=\sum_{i=1}^{k} x_{i}
$$

$\tilde{x}$ is the reconstructed denoising signal, and the determination of effective singular components (SCs) has always been a hot issue and difficult problem in the application of singular value decomposition.

\section{FIC-SVD method}

\subsection{Proposed indicator FIC}

Most of the indicators proposed in the previous literature were developed in the fields of statistics or information theory (e.g. kurtosis ${ }^{[8-9]}$ and entropy ${ }^{[10]}$ ) and recently developed sparse metrics, such as norm ${ }^{[11]}$ and Gini index ${ }^{[12]}$. They mainly focus on the general statistical distribution of signals and ignore the specific characteristics of mechanical signals, so it may not be possible to select the SCs with the most information from the perspective of diagnosis and prognosis. In view of the above problems, this paper proposes a new index FIC for the SCs selection and signal denoising.

According to the amplitude filtering characteristics of singular value decomposition proposed by Guo et al. ${ }^{[13]}$, the larger the amplitude of the frequency component is, the more fault information the corresponding singular component contains. That is, the order of the singular value depends on the corresponding frequency component signal amplitude. The specific relationship is listed in formula (7):

$$
\left(\sigma_{2 \mathrm{i}-1}^{2}+\sigma_{2 \mathrm{i}}^{2}\right) \propto a_{i}^{2}
$$

where $\sigma_{2 i-1}$ and $\sigma_{2 i}$ are the two singular values corresponding to the singular components, and $a_{i}$ is the corresponding amplitude.

When the singular component contains fault information (the singular component is valid), the envelope spectrum can detect the fault. That is, the one-time fault frequency amplitude and two times the fault frequency amplitude can be read out in the envelope spectrum, and the information amount of the singular component can be determined based on the magnitude of its amplitude. However, the monitorability of the fault characteristics depends not only on the magnitude of the fault itself, but also on the average magnitude. Therefore, it is more reasonable to use the ratio of the two as the measurement standard, that is, the proposed index FIC.

$$
F I C=\frac{E\left(f_{r}\right)+E\left(f_{2 r}\right)}{E M}
$$

where $E\left(f_{r}\right)$ and $E\left(f_{2 r}\right)$ are the one-time fault frequency amplitude and two times the fault frequency amplitude in the singular component envelope spectrum, respectively, and $E M$ is the average amplitude of the envelope spectrum.

\subsection{FIC-SVD process}

By combining the SVD algorithm with the indicators mentioned above, a new singular value decomposition algorithm, namely FIC-SVD, is proposed in this paper. The specific steps are described as follows, and the flowchart is shown in Figure 2.

(1) Construct the Hankel matrix of the original signal. When choosing an appropriate value of $m$, there is a compromise between computational efficiency and decomposition performance. In the 
literature [6], according to the simulation analysis and industrial data, it is tested that the selection of $\mathrm{m}$ values between 30 and 50 is satisfactory for different applications. In this paper, the value of $\mathrm{m}$ is chosen to be 40 empirically.

(2) Apply SVD to Hankel matrix, and obtain SCs by direct method, namely $x_{1}, x_{2}, \ldots, x_{m}$.

(3) Calculate the equal amplitude of the fault frequency of the SCs envelope spectrum to obtain the index FIC.

(4) Sort and select SCs according to FIC values.

(5) Add the reserved SCs to obtain a reconstructed signal, so as to achieve noise reduction and fault diagnosis.

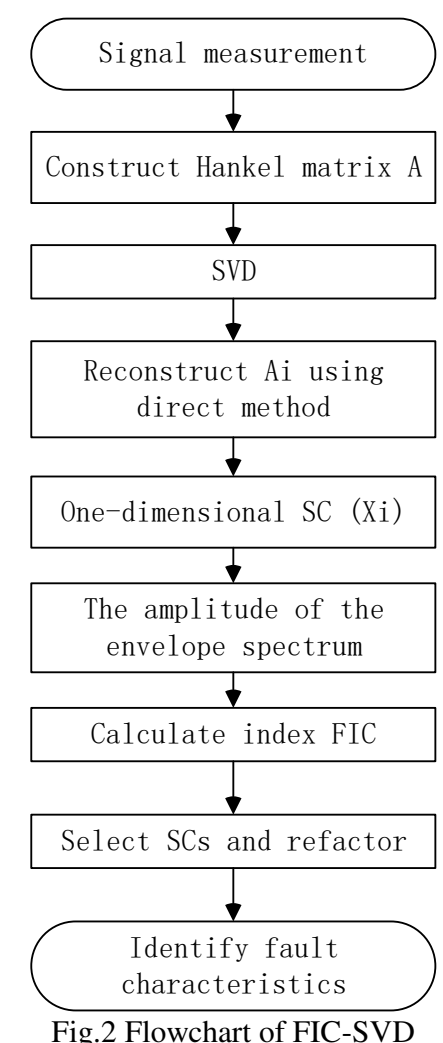

\section{Simulation signal analysis}

\subsection{Bearing fault simulation model}

In order to verify the correctness and effectiveness of the proposed method FIC-SVD, firstly, the bearing fault simulation signal constructed as in equation (9) is studied, analyzed and compared. In order to make the model more realistic and closer to the vibration signal measured in the industrial environment, the constructed signal contains both fault signals, harmonics and other background noises generated by the gear meshing.

$$
\begin{aligned}
x(t) & =\sum_{n} A_{n} \cos \left(2 \pi n f_{\alpha} t+\alpha_{n}\right)+\sum_{n} B_{n} \cos \left(2 \pi n T f_{\beta} t+\beta_{n}\right) \\
& +\sum_{i} D_{i} e^{-\varepsilon\left(t-i T_{d}-\tau_{i}\right)} \sin \left(2 \pi f_{n}\left(t-i T_{d}-\tau_{i}\right)\right)+n(t)
\end{aligned}
$$

where first term represents the vibration of the rotor and the shaft, where $f_{\alpha}$ represents the fundamental rotation frequency, and $A_{n}$ and $\alpha_{n}$ are the amplitude and initial phase of the nth harmonic, respectively. The second term represents the vibration caused by the normal gear meshing, where $f_{\beta}$ represents the basic rotation frequency, T is the number of gear teeth, and $B_{n}$ and $\beta_{n}$ are the amplitude and initial phase of the nth meshing frequency, respectively. The third term represents the 
pulse excited by the mechanical defect, where $D_{i}$ is the amplitude of the i-th pulse, $T_{d}$ specifies the time interval between two adjacent pulses, $\tau_{i}$ is a random variable to simulate the sliding effect, and $\varepsilon$ is the attenuation coefficient. The last term $n(t)$ is white noise.

The time-domain waveform corresponding to each item is shown in Figure 3, and the time-domain waveform and envelope spectrum of the original simulation signal are shown in Figure 4.

Table 1 Parameter values

\begin{tabular}{cccccccc}
\hline$A_{1}$ & $f_{\alpha}$ & $B_{1}$ & $B_{2}$ & $f_{\beta}$ & $D_{i}$ & $T_{d}$ & $f_{n}$ \\
\hline 0.1 & 10 & 0.2 & 0.08 & 10 & 0.3 & $1 / 30$ & 2400 \\
\hline
\end{tabular}
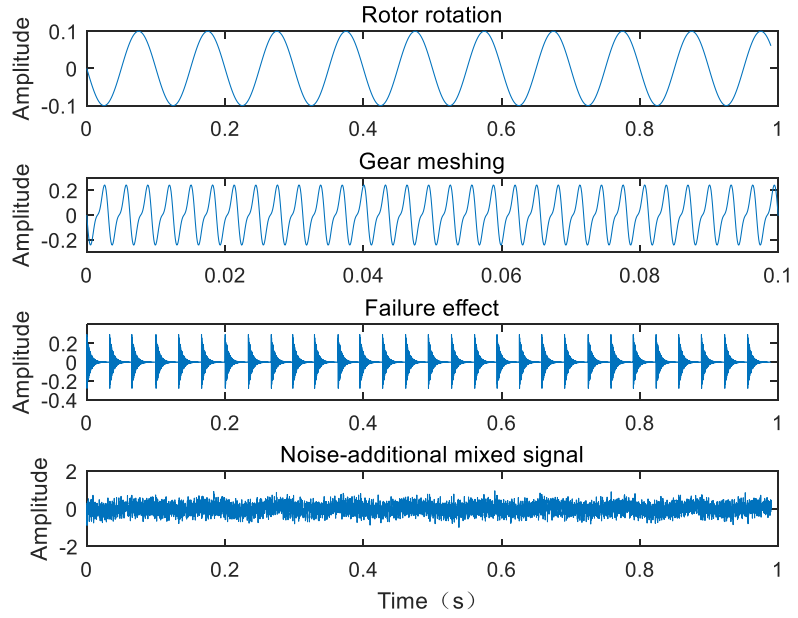

Fig.3 Components of the simulated signal
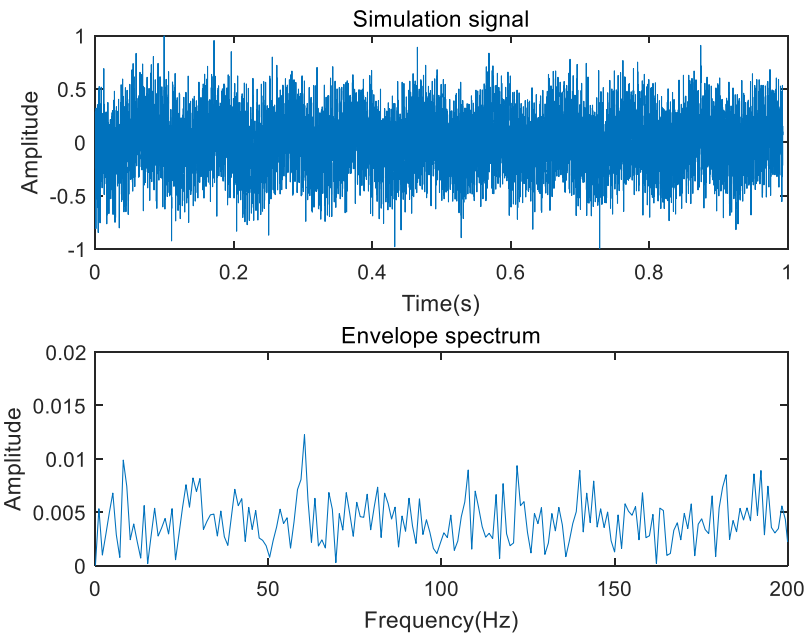

Fig.4 Time domain diagram and envelope diagram of simulation signal

\subsection{Comparisons of FIC-SVD and other methods}

First, wavelet transform is used to denoise the simulated signal. In this paper, the wavelet base is chosen as coif5, and the number of decomposition layers is 7 layers. Figure 5 is the time domain diagram and envelope diagram of the signal reconstructed by wavelet transform. It can be seen that the fault cannot be diagnosed well. 

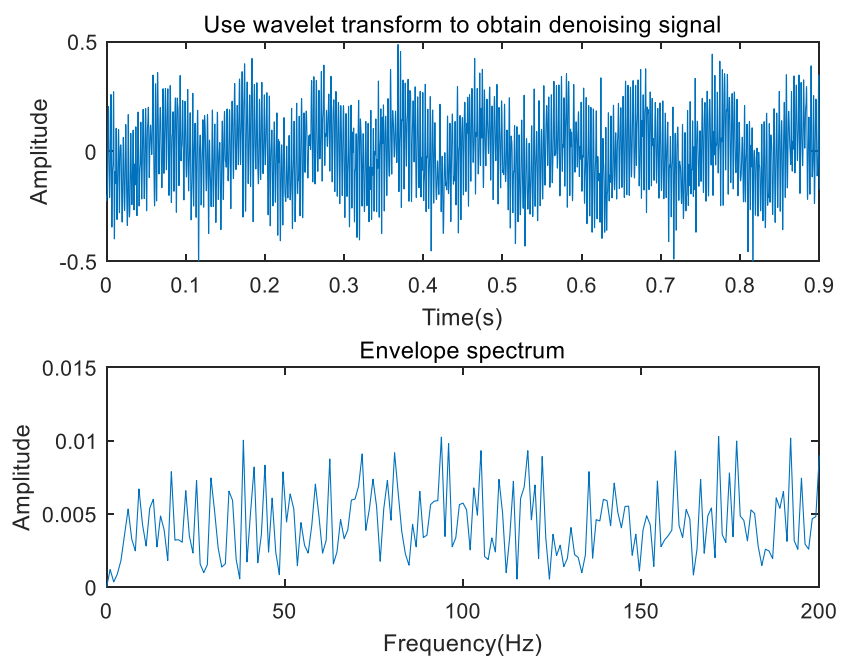

Fig.5 Time domain diagram and envelope diagram of wavelet transform

Secondly, the traditional DS-SVD method is used for the bearing fault simulation signal. The calculated singular values and their difference spectrum are shown in Figure 6. According to the traditional DS-SVD method, the threshold $\mathrm{K}$ is often determined as the peak value in the singular value difference spectrum (DS), and then the first k SCs are chosen to be retained to reconstruct the signal. It can be seen from Figure 6 that the DS has a clear peak at $\mathrm{k}=2$, so the noise reduction of the original signal is achieved by retaining the first two SCs.

Figure 7 shows the time-domain waveform diagram and envelope spectrum diagram of the reconstructed signal, but it can be seen that the reconstructed signal is almost stationary without any pulse signal. At the same time, no useful information about the fault can be obtained in the given envelope spectrum.
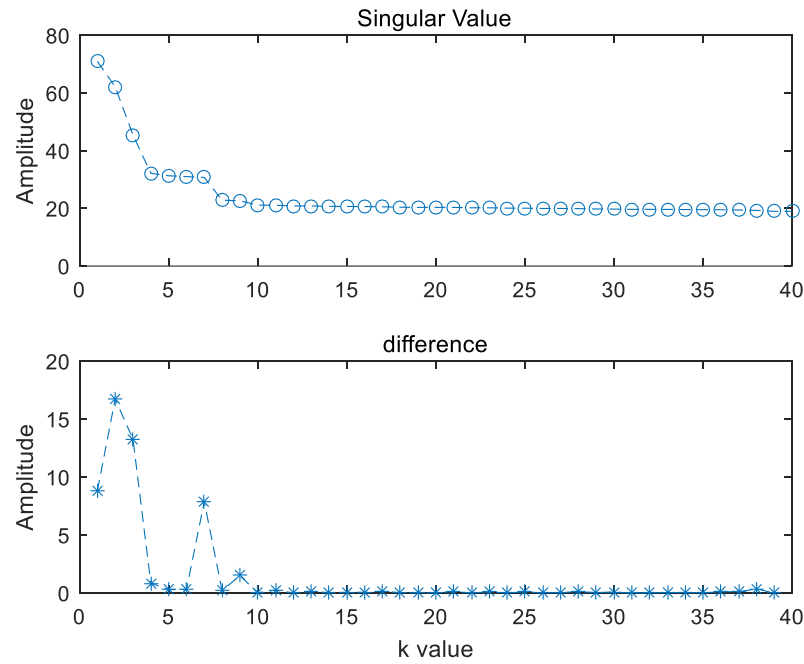

Fig.6 Singular value and difference diagram 

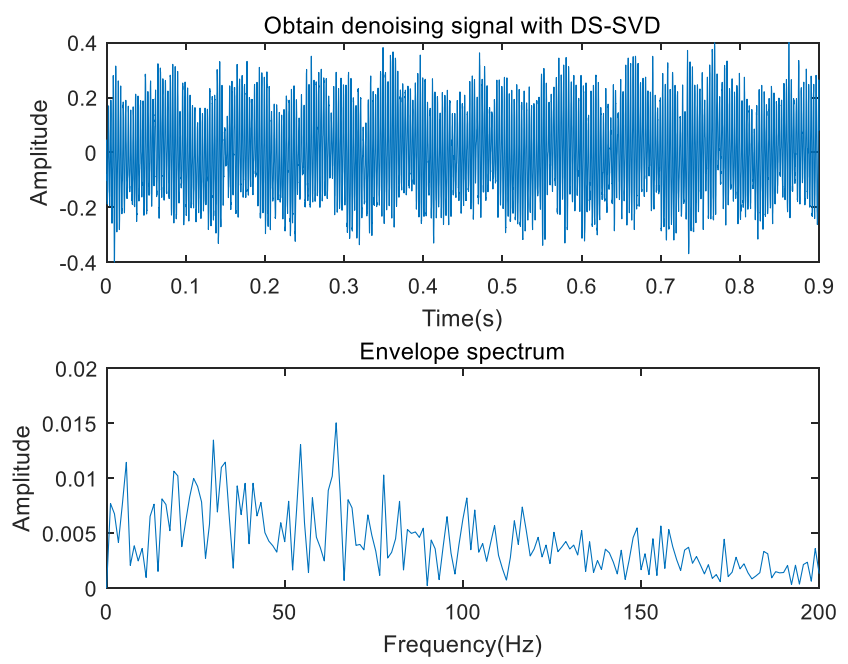

Fig.7 Time domain diagram and envelope diagram of DS-SVD

Figure 8 depicts an explanation of this phenomenon that traditional selection methods are highly correlated with singular values, and SCs with high energy are often selected. As shown in Figure 9 for gear meshing and rotor vibration, these items tend to have higher energy, so in the traditional method, these SCs are retained as reconstructed signals. However, in the context of diagnosis, these SCs rarely carry information related to the machine health, so they can be considered redundant. On the other hand, some SCs that contain diagnostic information, such as the 6th and 7th, are ignored and discarded because of their weak energy.

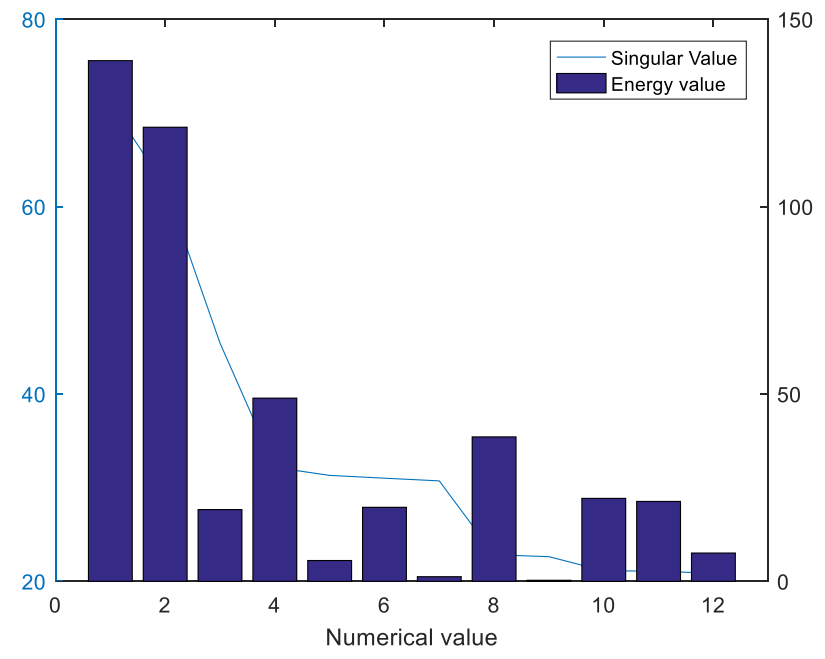

Fig.8 Relationship between singular value and energy

Aiming at the problem that the traditional method cannot well select the signal containing the diagnostic information, the FIC-SVD proposed in this paper is applied to the signal.

As shown in Figure 10, the FIC index value of each SC is calculated at first. It is not difficult to see from the figure that although the first two SCs have the largest energy, their diagnostic value is quite limited (the FIC value is small). It can also be observed that the FIC values of the 6th and 7th SCs are much larger than the other values, and these two SCs are derived from defective pulses, which have fault information from the perspective of diagnosis. Based on the above results, the 6th and 7th SCs are selected in this paper, and the denoising of the signal is applied through the addition of these two SCs.

Figure 11 shows the time-domain waveform diagram and envelope spectrum diagram of the noise reduction signal obtained using this method. It can be seen that using this method can effectively 
retain the diagnostic information caused by mechanical defects. Meanwhile, from the envelope spectrum of the noise reduction signal, the fault frequency and its harmonics can be clearly identified, and the magnitude is consistent with the theoretical calculation value of the simulation data. These evidences all show that the simulated rolling bearing has mechanical defects, and the proposed method is effective.
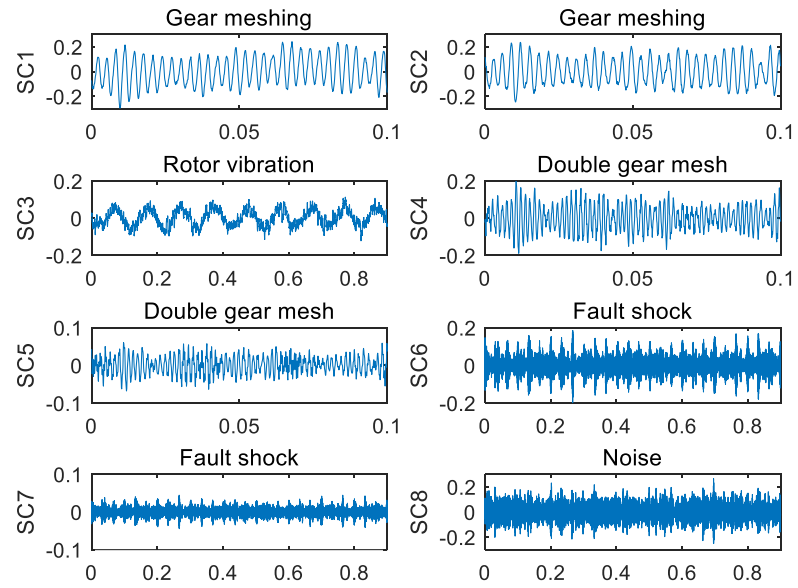

Fig.9 The first eight SCs of SVD

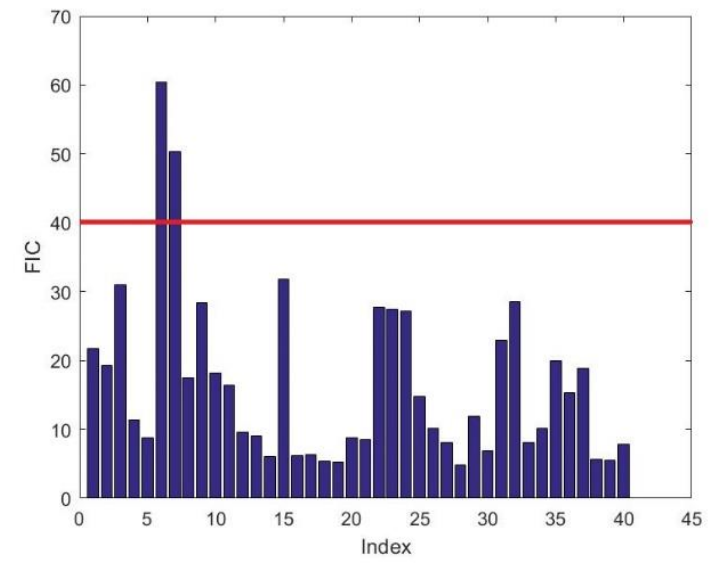

Fig.10 Index FIC of SCs
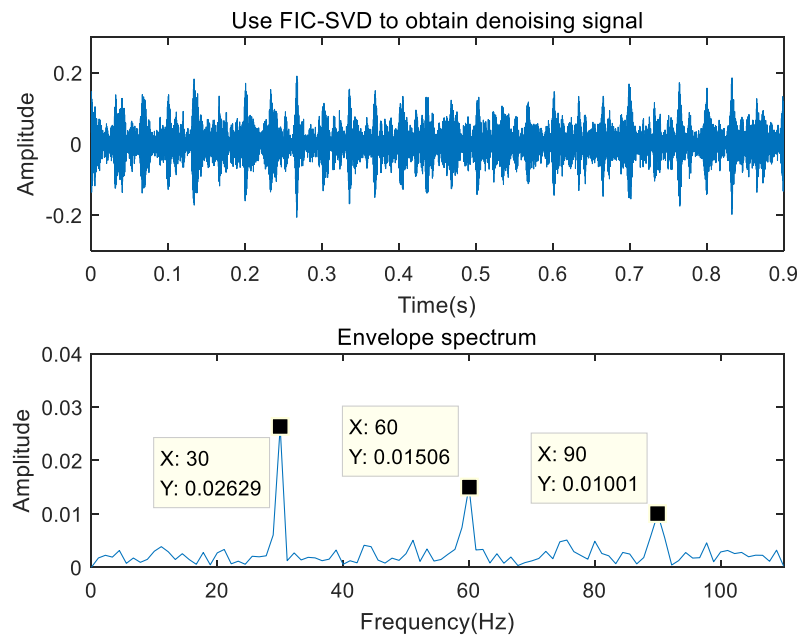

Fig.11 Time domain diagram and envelope diagram of FIC-SVD 


\section{Experimental data analysis}

\subsection{Case 1}

The bearing data of Western Reserve University is used for the analysis. The test bench and acquisition equipment are shown in Figure 12. The known bearing model is 6205-2RS. Its inner diameter is $25 \mathrm{~mm}$, outer diameter is $52 \mathrm{~mm}$, pitch diameter is $44.2 \mathrm{~mm}$, thickness is $15 \mathrm{~mm}$, rolling element diameter is $8.182 \mathrm{~mm}$, number of rolling elements is 9 , motor speed is $1797 \mathrm{r} / \mathrm{min}$, sampling frequency is $f_{s}=48000 \mathrm{~Hz}$. The damage to the outer ring of the bearing is a single-point damage made by EDM. The diameter of the damage is $0.1778 \mathrm{~mm}$ and it is located in the six o'clock direction.

According to the proposed method, the experimental data is decomposed by SVD firstly, and then the FIC value of each obtained singular component is calculated, as shown in Figure 13. The first, fourteenth, and twenty-fourth SCs have the largest FIC values, so the three singular components are selected to be added to construct the noise reduction signal.

Figure 14 shows the time-domain waveform diagram and envelope spectrum diagram of the noise reduction signal. It can be seen that the FIC-SVD method can effectively detect the fault information. From the envelope spectrum of the noise reduction signal, the fault frequency and its harmonics can also be clearly identified. These evidences show that the proposed method can be used in experimental bearing failure signals, and the diagnosis results are correct and effective.

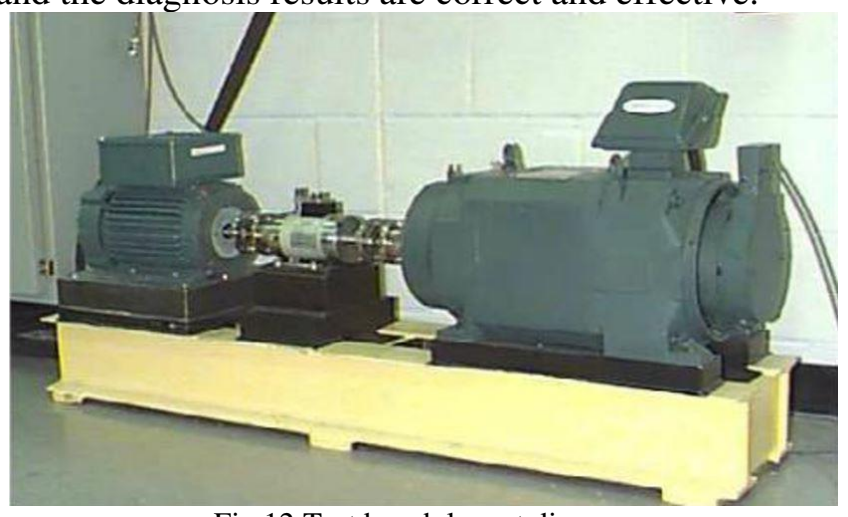

Fig.12 Test bench layout diagram

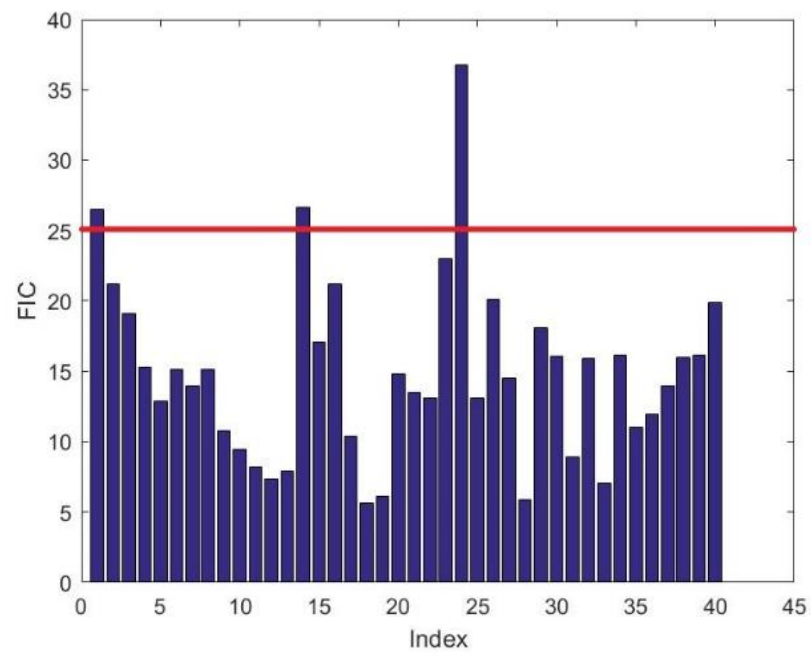

Fig.13 Indicator FIC of SCs 

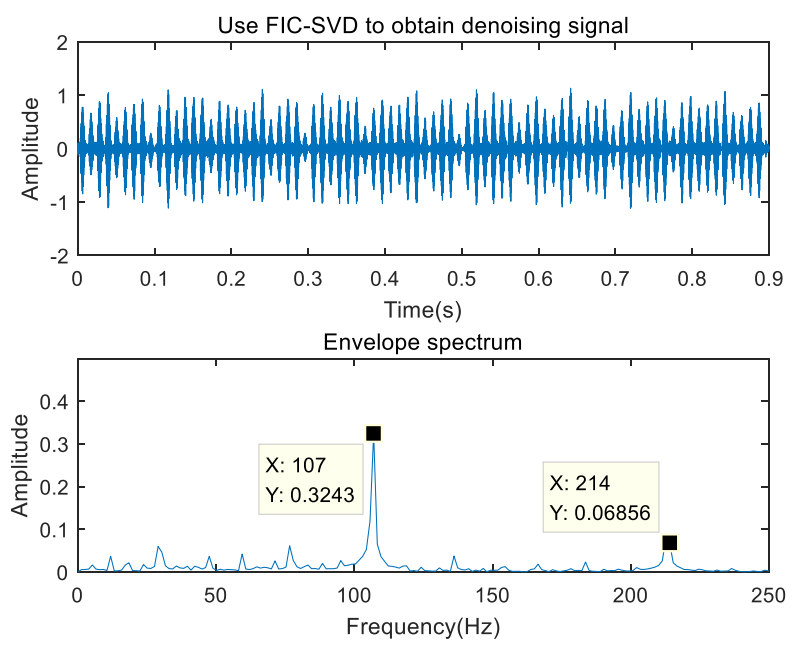

Fig.14 Time domain diagram and envelope diagram of FIC-SVD

\subsection{Case 2}

The Cincinnati whole life data is used for analysis, and the layout of the test bench is shown in Figure 15. The bearing model is Rexnord ZA-2115, the pitch diameter is $71.5 \mathrm{~mm}$, the rolling element diameter is $8.4 \mathrm{~mm}$, the number of rolling elements is 16 , the motor speed is $2000 \mathrm{r} / \mathrm{min}$, and the sampling frequency is $f_{s}=20000 \mathrm{~Hz}$.

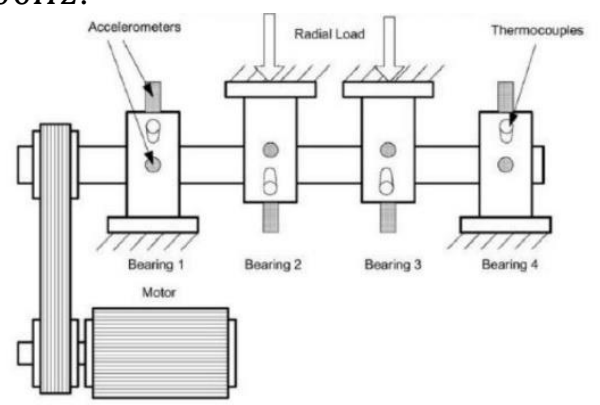

Fig.15 Test bench layout diagram

Similarly, singular value decomposition is used for the signal and the FIC value of the singular component is calculated, as shown in Figure 16. It can be seen that the FIC value of the first SC is the largest, so this singular component is retained to construct the noise reduction signal. The time-domain waveform diagram and envelope spectrum diagram are shown in Figure 17. Obvious fault frequencies and their harmonics can be seen. The proposed method is suitable for this case.

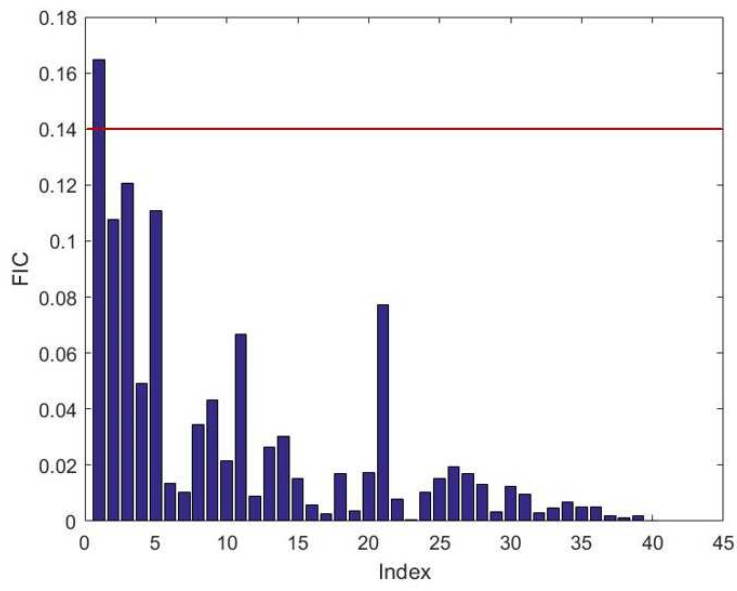

Fig.16 Indicator FIC of SCs 

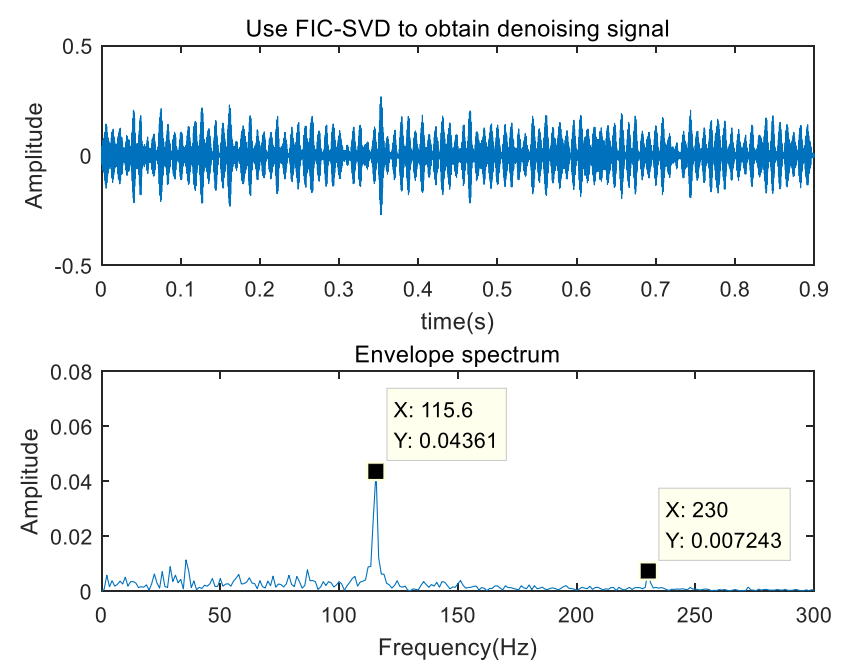

Fig.17 Time domain diagram and envelope diagram of FIC-SVD

\section{Conclusion}

This paper proposes a new weak feature enhancement method based on singular value decomposition. That is, the original signal is first decomposed by SVD to obtain a series of singular components. And then the components to be retained are selected by calculating the corresponding FIC values. At last the denoising signal is reconstructed after the addition.

The analysis results of simulation and experimental signals show that the proposed FIC-SVD method is an effective description of bearing fault behavior and can be used to select fault features with relatively low energy.

Compared with the previous SVD noise reduction method, this method breaks through the limitations of previous noise reduction strategies, redefines the concept of "information" in the context of rotating machinery, and highlights the characteristics of failures.

\section{Funding}

This research is funded by the National Natural Science Foundation of China ( Grant Nos. 52075008 ).

\section{Conflicts of interest/Competing interests}

The content of this paper does not involve any conflict of interest.

\section{Availability of data and material}

The data used in this paper are all owned by the lab of the research group. As the research is still continuing, the data involved in this paper is not publicly available.

\section{Code availability}

The algorithm involved in this paper is still being studied by the research group, so it is not publicly disclosed.

\section{Ethics approval}

The team affirmed that all the research was original research of the research group without any bad behavior.

\section{Consent to participate}

The methods involved in this paper are agreed to be shared. 


\section{Consent for publication}

The content of this thesis is agreed to be published in the journal.

\section{Authors' contributions}

M, X. S analyzed the data; L, L. C. and J.F. H provided guidance and recommendations for the research. M, X. S contributed to the contents and writing of the manuscript. All authors have read and approved the final manuscript.

\section{Acknowledgment}

This work is supported by the National Natural Science Foundation of China under Grant No. 52075008. The authors are grateful to the editors and anonymous reviewers for their helpful comments and constructive suggestions.

\section{References}

[1] Tandon N, Choudhury A. A review of vibration and acoustic meas - urement methods for the detection of defects in rolling element bearings [J]. Tribol Int 1999( 32) : 469-480.

[2] Liu Yuanxing. Analysis on the repair of rolling bearings[J]. Heilongjiang Science,2014(7):248248.

[3] Chang Li, Zhao Ruojun. Research on Fault Diagnosis of Fan Bearing Based on Acoustic Emission[J]. Instrument Technology and Sensor,2017(09):109-113.

[4] R. Golafshan, K. Yuce Sanliturk, SVD and Hankel matrix based de-noising approach for ball bearing fault detection and its assessment using artificial faults, Mech. Syst. Signal Process. 70-71 (2016) 36-50.

[5] W. X. Yang, P. W Tse., Development of an advanced noise reduction method for vibration analysis based on singular value decomposition, NDT\&E International 36 (6) (2003) 419432.

[6] M. Zhao, X. Jia, A novel strategy for signal denoising using reweighted SVD and its applications to weak fault feature enhancement of rotating machinery, Mech. Syst. Sig. Process. 94(2017) 129-147.

[7] Y. X. Zhang, X. L Wang., SH. Zhang, et al, Rolling element bearing fault diagnosis based on singular value decomposition and correlated kurtosis, Journal of vibration and shock 33 (11) (2014) 167-171.

[8] P. Borghesani, P. Pennacchi, S. Chatterton, The relationship between kurtosis- and envelopebased indexes for the diagnostic of rolling element bearings, Mech. Syst. Signal Process. 43 (2014) 25-43.

[9] Y. Lei, Z. He, Y. Zi, EEMD method and WNN for fault diagnosis of locomotive roller bearings, Expert Syst. Appl. 38 (2011) 7334-7341.

[10] J. Lin, L. Qu, Feature extraction based on Morlet wavelet and its application for mechanical diagnosis, J. Sound Vib. 234 (2000) 135-148.

[11] P.W. Tse, D. Wang, The design of a new sparsogram for fast bearing fault diagnosis: part 1 of the two related manuscripts that have a joint title as "Two automatic vibration-based fault diagnostic methods using the novel sparsity measurement - Parts 1 and 2", Mech. Syst. Signal Process. 40 (2013) 499-519.

[12] D. Zonoobi, A.A. Kassim, Y.V. Venkatesh, Gini index as sparsity measure for signal reconstruction from compressive samples, IEEE J. Sel. Topics Signal Process. 5 (2011) $927-$ 932.

[13] M. Guo, W. Li, Q. Yang, X. Zhao, Y. Tang, Amplitude Filtering characteristics of Singular Value Decomposition and Its Application to Fault Diagnosis of Rotating Machinery, Measurement (2019). 